\title{
Single file diffusion in macroscopic Wigner rings
}

\author{
Gwennou Coupier, ${ }^{1}$ Michel Saint Jean, ${ }^{1, \text { * }}$ and Claudine Guthmann ${ }^{1}$ \\ ${ }^{1}$ Laboratoire Matière et Systèmes Complexes, UMR $705^{r}$ CNRS 8 \\ Université Paris 7 - 140 rue de Lourmel, F-75015 Paris, France
}

(Dated: October 8, 2018)

\begin{abstract}
The single file diffusion in a circular channel of millimetric charged balls is studied. The evolution in time of the mean square displacement is shown to be subdiffusive, but slower than the power-like $t^{1 / 2}$ behavior observed in circular colloidal systems or predicted in one-dimensional infinite systems.

PACS numbers: 05.40.-a
\end{abstract}

\section{INTRODUCTION}

Diffusion of particles in a single channel where the mutual exchanges are forbidden is known as single file diffusion (SFD). This physical situation is encountered in various fields, for instance $1 \mathrm{D}$ hopping conductivity, ion transport in biological membranes or molecules channeling in zeolithes. In these geometries with low dimensionality, the particles in repulsive interaction are not able to cross each other, and the particles remain correlated even at long times.

Such systems were generally modeled by an infinite set of particles with hard interaction and diffusing on a line [1, 2]: the mean square displacement $\Delta x^{2}$ has been proved to grow at long times as $t^{1 / 2}$. More recently, it was shown that this behavior is also encountered in overdamped systems with arbitrary repulsive interactions, providing the correlation length between the particles is of finite range [3]. On the other hand, although the main theoretical results for single file diffusion were obtained many years ago, experiments displaying such subdiffusive behavior are lacking and the obtained results are often conflicting. For instance, NMR studies 4, 5] and quasi elastic neutron scattering experiments [4, 6] on organic molecules in porous materials either conclude to subdiffusive transport [4, 5] or to classical diffusion [4, 6], with apparently the same experimental conditions. More recently, the movement of colloidal particles in a circular channel has been studied. This channel is obtained by photolithography [7, 8] or optical tweezer circular trapping [9]. While classical diffusion is always observed for short times, the long time behavior is not as well clearly analyzed. Some authors suggest it grows as $t^{1 / 2}$ like theoretical SFD processes for infinite systems [7, 9] whereas others explain this subdiffusion by hydrodynamic coupling effects [8]. On the other hand, the theoretical study made in Ref. 2] for a 1D line of hard-core interacting particles with periodic boundary conditions shows that the mean square displacement grows linearly, as for a free diffusion.

In order to rule out these ambiguities, we present here

*Electronic address: michel.saintjean@paris7.jussieu.fr

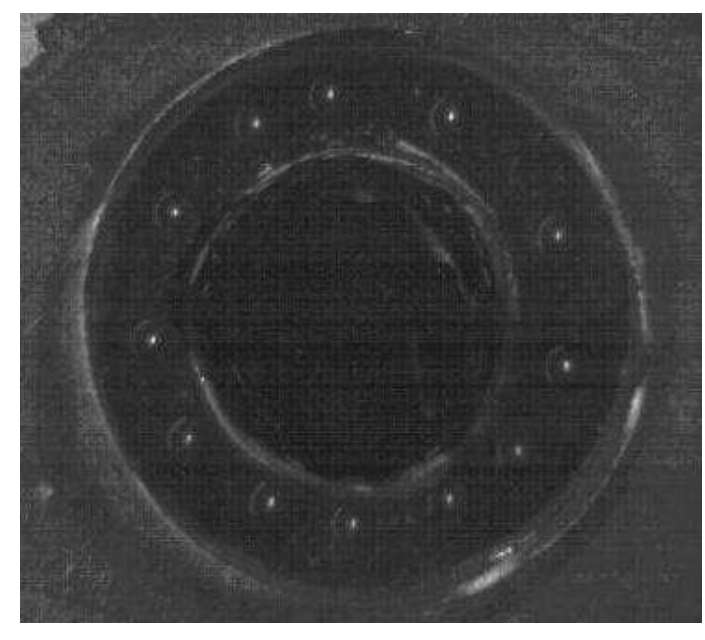

FIG. 1: Photography of the experimental circular channel with $N=12$ balls inside.

the diffusion of macroscopic charged metallic balls electrostatically interacting and moving in a circular channel whose width forbids any crossing, while a mechanical shaking induces an effective temperature. This system presents many advantages : it gives the opportunity to study the diffusion at very long times, it allows to suppress any hydrodynamic effect and, if necessary, to tune the interacting forces. In this experiment, the inter-particle interaction is similar to $K_{0}$ inter-vortices interaction in superconductors 10]. This interaction differs from the dipolar interaction of colloids or from the hard interaction introduced in many theories, thus we can evaluate the influence of the interaction characteristics on the SFD behaviors. Note that no hard-core collisions are observed in our system.

Our main result is to exhibit subdiffusive behavior slower than the $t^{1 / 2}$ behavior predicted by theory and mentioned in colloidal systems.

In Sec.II we present the experimental set-up and prove its ability to describe diffusion processes by studying two basic cases. To identify the effects of the circular confinement on the diffusion in order to distinguish them from the inter-particle interaction contributions and to determine the best conditions to obtain the required 1D movement, we have studied the diffusion of a single ball in the channel. The obtained results are reported in Sec. III 
Sec. IV] is devoted to the diffusion of $N=12$ and 16 interacting particles and to the comparison with the behaviors previously presented in literature, in particular those obtained with colloids.

\section{EXPERIMENT VALIDATION}

In this experiment, millimetric stainless steel balls (of radius $R=0.4 \mathrm{~mm}$ and weight $m=2.15 \mathrm{mg}$ ) are located on the doped-silicon bottom electrode of an horizontal plane condenser (of height $h=1.5 \mathrm{~mm}$ ). A metallic frame intercalated between the two electrodes and in contact with the bottom one confines the balls in a circular channel. Its external diameter and its width are respectively $10 \mathrm{~mm}$ and $2 \mathrm{~mm}$ (Fig 1). This forbids any crossing between the balls. In order to charge the balls, a tunable voltage $V_{c}$ of about $1 \mathrm{kV}$ is applied to the top electrode. We then get a system where the interaction between the balls as well as the confining potential are easily adjusted. The interaction potential $V(r)$ between two balls has been shown to be well described through a modified Bessel function of the second kind : $V(r)=A K_{0}(r / \lambda)$ where the screening length $\lambda$ is about $0.3 h=0.5 \mathrm{~mm}$ and independent from $V_{c}$, as discussed in details in Ref. 10]. Direct capture of the position of the balls is made by a camera placed above the experimental device. The typical time between two snapshots is selected between 15 $\mathrm{ms}$ and $150 \mathrm{~ms}$ and series of 10,000 images were recorded. The individual trajectories of these particles are thus directly determined over very long times (comparing with the typical relaxation time that will be determined in the following).

To introduce the thermal noise, the whole cell is fixed on loudspeakers supplied by a white noise voltage. Thanks to the friction between the bottom electrode and the balls, a erratic and non spatially correlated movement is conveyed to the latter. It has been previously shown that the ensemble of balls obey Boltzmann statistics, where the shaking amplitude stands for the temperature 11]. The effective temperature is thus determined in situ by measuring the mean square position of a single ball confined by a circular frame.

The diffusion of the particles is characterized by the evolution with time of their mean square displacements (m.s.d.) given by, if the considered coordinate is $x$ :

$$
\Delta x^{2}(t)=\left\langle(x(t)-\langle x(t)\rangle)^{2}\right\rangle,
$$

where the brackets \langle\rangle denote ensemble averaging. In practice, we get from the trajectory of one ball a set of statistically independent trajectories by shifting the time origin. We emphasize that such a method implies unavoidable averaging over the distribution of initial conditions, which can lead to mean square displacements slightly different than the usual ones (see the appendix). Furthermore, when considering the orthoradial coordinate $\theta$ (in radians) we will consider the cumulated angle and not the modulo $2 \pi$ angle, since we want to con-
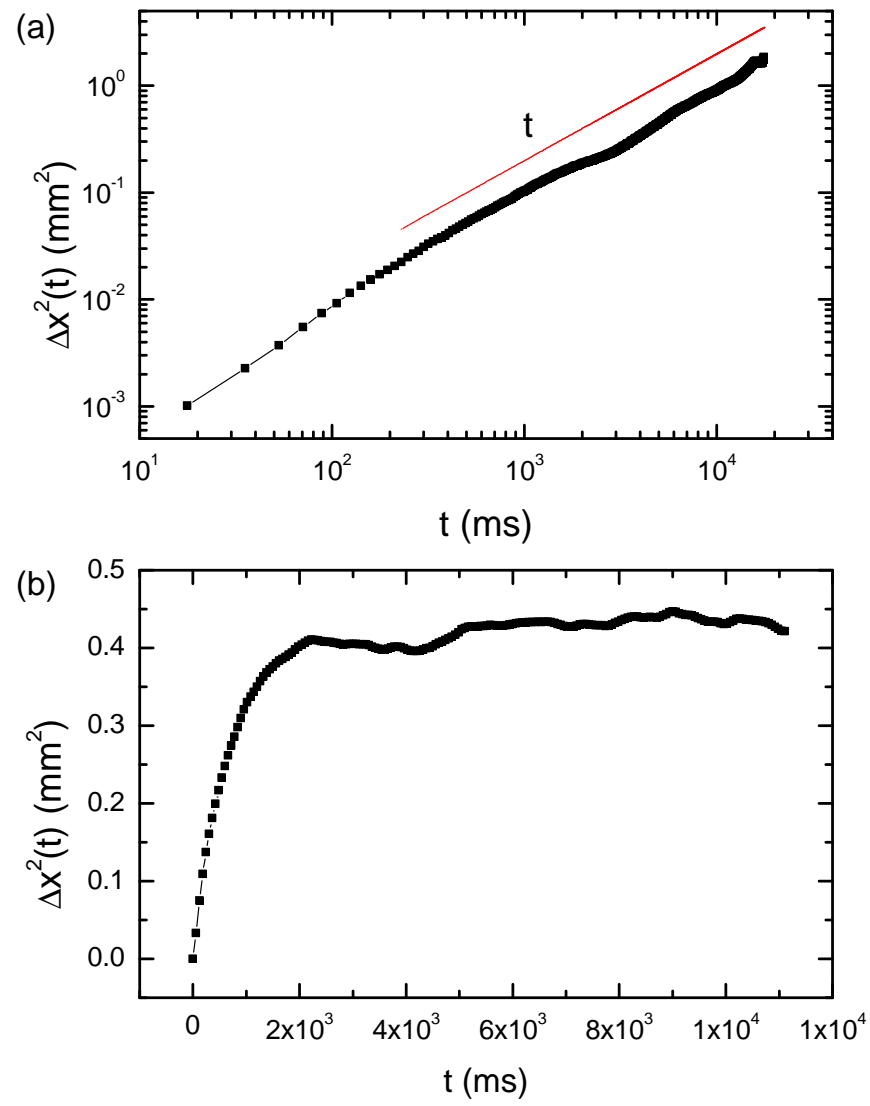

FIG. 2: (a) Typical mean square displacement of a free ball (log scale) ; (b) typical mean square displacement of a ball trapped in a well constituted by a circular frame of radius 5 $\mathrm{mm}$. The applied voltage $V_{c}$ is $1000 \mathrm{~V}$.

sider the ring as an easy realization of a $1 \mathrm{D}$ line. As in Ref. 7, 8, 9], the total orthoradial displacement of a ball is taken into account, without any substraction of a mean displacement of the system.

In order to evaluate the ability of our experimental setup to explore diffusion processes, two simple cases for which the diffusion behaviors are well established have been studied.

The free diffusion of a ball moving on the bottom electrode without any confinement and any applied electrostatic potential was first studied. Whatever the effective temperature, the m.s.d. $\Delta x^{2}(t), x$ being the ball abscissa, presents the same typical time variation, presented on Fig. 2(a). This m.s.d. varies linearly with time at long times, whereas the short time behavior exhibits a $t^{\beta}$ power increase with $1<\beta<2$. These behaviors are in agreement with a free diffusion described by the classical Langevin equation which predicts $\Delta x^{2}(t) \simeq 2 D t$ at long times where $D$ is the diffusion constant. Since the temperature is independently measured, the effective damping coefficient $\gamma$ can be determined from the fluctuation-dissipation theorem $m \gamma D=k_{B} T$. The related relaxation time $\tau_{R}=1 / \gamma$ which marks the crossover between the short and long time regimes is found to be 
equal to about $100 \mathrm{~ms}$, which is coherent with a rough estimation on the curve of Fig. 2(a).

This value which is quite close to the smallest snapshot time explains why the theoretical behavior in $t^{2}$ predicted for very short times $\left(t \ll \tau_{R}=1 / \gamma\right)$ may not be observed, the behavior observed at short times corresponding actually to the progressive transition between a $t^{2}$ regime to a linear one (this analyze is confirmed by the measured mean square velocity which is always constant in time as expected in the Langevin theory for $t>\tau_{R}$ ).

The second test was to follow the diffusion of a particle confined in a well created by a circular frame (of radius $5 \mathrm{~mm}$ ). In such a case, the ball moves in a parabolic confining potential whose stiffness $K$ can be determined from the radial distribution of the ball [11]. Fig. 2(b) presents a typical radial m.s.d. of a ball in such potential. After a rapid increase, it reaches a constant value which varies linearly with the effective temperature as expected. These behaviors are those predicted in a Langevin formalism in which a parabolic potential models the confinement. At short times $\left(t<(K / m)^{1 / 2}\right)$, the ball begins to follow a free diffusion since the particle has no time to explore the whole confining potential whereas at long times $\left(t>(K / m)^{1 / 2}\right)$ the experimental m.s.d. is dependent on the confinement and reaches $2 k_{B} T / K$ (see the appendix). In the same time, the angular m.s.d grows linearly with time, which characterizes a free diffusion along the orthoradial direction.

The accordance between the theoretical behavior and the observed one confirms the ability of our experimental set-up to give relevant and self coherent parameters about particle diffusion and that the Langevin formalism describing Brownian motion can be used.

\section{DIFFUSION OF A SINGLE BALL IN A CIRCULAR CHANNEL.}

In our experiments, the interacting balls move in a electrostatic confining potential which looks like a circular gutter. In order to distinguish possible effects due to this channel geometry from those resulting from the inter-particle interaction, we have preliminary studied the diffusion of a single ball in such a circular channel. In particular, the radial confining potential has been studied for different applied electrostatic potentials in order to get the best opportunities for a quasi $1 \mathrm{D}$ movement. In the same time, the coupling of the small radial displacement with the orthoradial movement inherent to the circular movement has also been evaluated.

At low temperatures, the ball oscillates radially with a slow orthoradial drift. As the temperature increases, the orthoradial displacements grow, the ball runs over the whole channel with long free jumps between two sequences of radial oscillations. Note that no bounces on the channel walls have been observed whatever the temperature, the ball is only confined by the electrostatic potential. Its typical shape, determined from the distri-

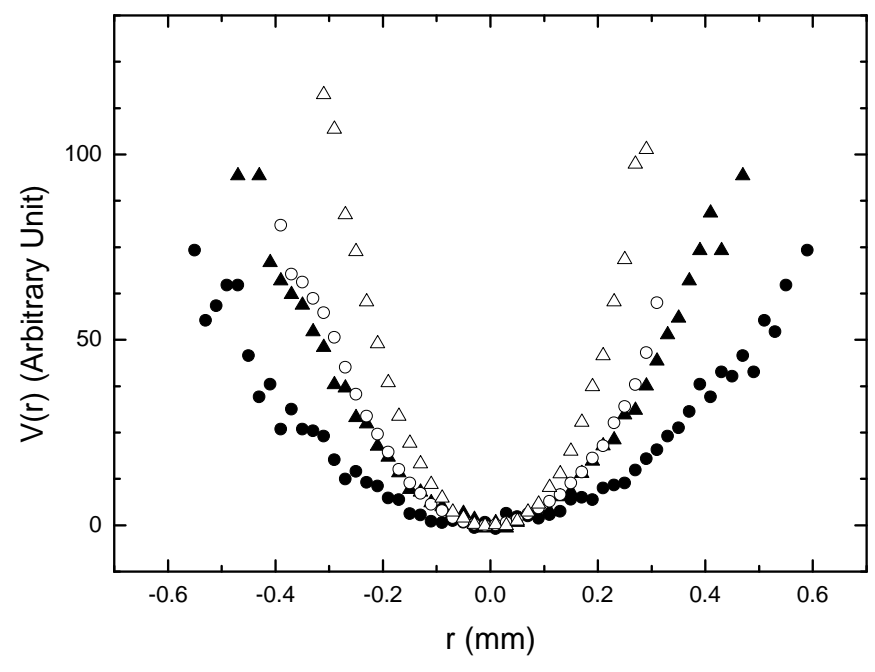

FIG. 3: Confining radial potential in the circular channel obtained from the radial distribution of the balls, for two different numbers of balls and two different applied voltage $V_{c}$ : 1 ball, $V_{c}=800 \mathrm{~V}(\bullet) ; 1$ ball, $V_{c}=1000 \mathrm{~V}(\boldsymbol{\Lambda}) ; 12$ balls, $V_{c}=800 \mathrm{~V}(\circ) ; 12$ balls, $V_{c}=1000 \mathrm{~V}(\Delta) . r$ is measured relatively to the mean radial position.

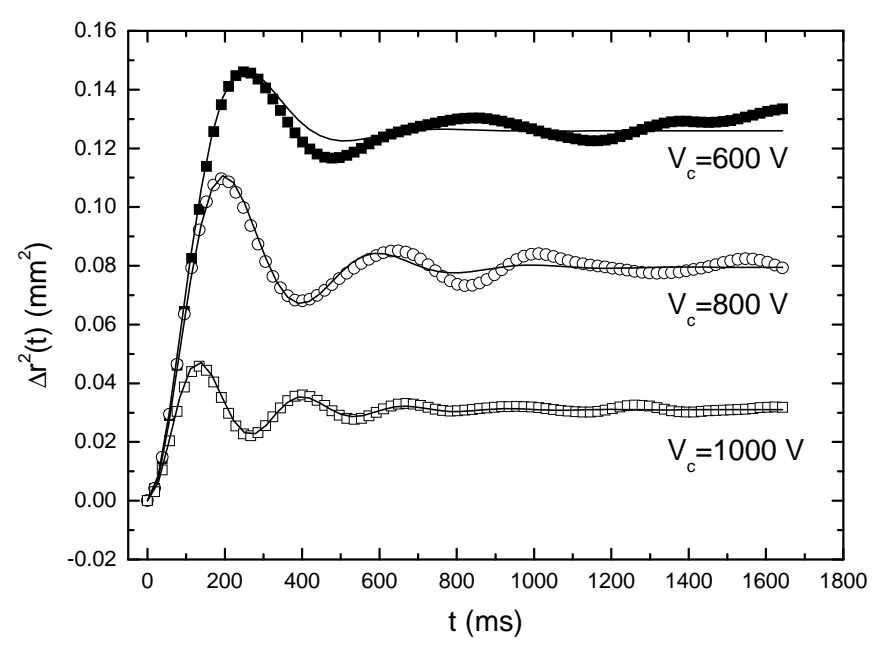

FIG. 4: Radial m.s.d. for a single ball in the circular channel for three different applied voltages. Solid lines show the fits to equation 2

bution of radial positions, is presented in Fig. 3. Whatever $V_{c}$, it is parabolic with a stiffness $K$ that increases with the applied voltage while the mean radial position remains unchanged. Let us indicate that for too small $V_{c}$, the electrostatic confinement looks more like the flat geometrical channel profile than a parabolic well. Therefore, we will work in the following with high $V_{c}(\simeq 1000 \mathrm{~V})$ in order to reinforce the 1D movement.

For a 1D parabolic potential, Langevin equation can be analytically solved and its characteristic coefficients determined by fits with the experimental data. The evolution with time of the radial m.s.d. of a single ball in the channel is shown on Fig. 4. At short times, it presents 
a rapid increase followed by small damped oscillations and finally reaches a constant value at long times. It is very well fitted by the theoretical expression obtained through Langevin formalism in the case of low friction (see the appendix),

$$
\Delta r^{2}(t)=2\left\langle\left\langle r_{0}^{2}\right\rangle\right\rangle\left[1-e^{-\gamma t / 2}\left(\cos (\omega t)+\frac{\gamma}{2 \omega} \sin (\omega t)\right)\right],
$$

where $\left\langle\left\langle r_{0}^{2}\right\rangle\right\rangle=k_{B} T / K$ is the mean square starting position, $\gamma$ the damping coefficient and $\omega=\sqrt{\omega_{0}^{2}-\gamma^{2} / 4}$ (with $\omega_{0}=K / m$ ) the effective frequency of the oscillations of the ball.

As expected, the larger the applied potential $V_{c}$ is, the better the fits are. The frequency $\omega$, which is temperature-independent and equal to about $20 \mathrm{~Hz}$ within the explored $V_{c}$ range, is in accordance with the independently measured frequency obtained from the Fourier transform of the ball trajectory, which confirms the validity of the use of the Langevin equation. The $K$ values determined from these frequencies are in perfect agreement with those evaluated from the radial distribution variance and varies as $V_{c}^{2}$ as expected from the determination of the interaction between a ball and the wall [10].

From the fits, the damping coefficient $\gamma$ is found to be independent from $V_{c}$ and equal to about $10 \mathrm{~s}^{-1}$. This value is of the same order as the one for free diffusion, which indicates that the relaxation time $\tau_{R}=\gamma^{-1}$ results uniquely in the case of a unique ball from the friction process on the bottom electrode. Within the temperature range, $\gamma$ is slightly temperature-dependent but, because of the respective range of $\omega_{0}^{2}$ and $\gamma^{2} / 4$, this dependence is not significant for $\omega$.

Note that the main feature of the presented fits is that the time $\tau_{R}$, which is an important time of reference when analyzing the orthoradial movement, can be precisely determined for each experiment.

Another point is the coupling between the orthoradial and the radial movement in the circular channel. The variations of the angular m.s.d. for $V_{c}=1000 \mathrm{~V}$ is presented in Fig. 7 The long time $\left(t \gg \tau_{R}\right)$ behavior is linear, which indicates a free orthoradial diffusion. This behavior is observed whatever the temperature. Note the oscillations of the radial m.s.d are not observed here, which strongly evidences that the orthoradial and radial movements are independent. This was suggested by the independence of $K$ with the effective temperature, because otherwise the effective stiffness would depend on the temperature through the orthoradial velocity. Moreover this angular free diffusion time dependence unambiguously confirms that the ball is only confined by the electrostatic potential and that the balls are not bouncing on the walls, otherwise different dependencies would be found [12].

Finally, from the measurement of the diffusion constant of the orthoradial movement and the measurement of the damping coefficient through the independent

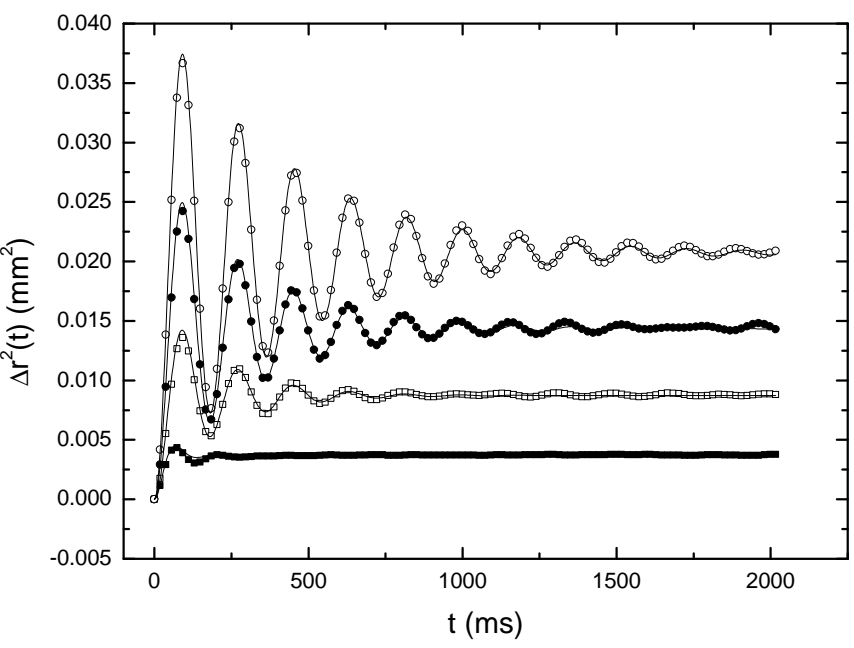

FIG. 5: Radial m.s.d of a ball in a 12-ball ring, with $V_{c}=1000$ $\mathrm{V}$ and $T=3.5 \times 10^{11} \mathrm{~K}(\mathbf{\square}), 7.5 \times 10^{11} \mathrm{~K}(\square), 11.5 \times 10^{11} \mathrm{~K}$ $(\bullet), 17.5 \times 10^{11} \mathrm{~K}(\circ)$.Solid lines shows the fit to equation 2

study of the radial movement, one can check that the fluctuation-dissipation relation is obeyed, which proves that the thermal equilibrium is reached (as also shown in Ref. [1] ]) and justifies the use of the latter relation to determine any missing parameters in a given experiment.

Thus the movement of one particle trapped in the circular channel is twofold. Along the radial direction it is a $1 \mathrm{D}$ diffusion in a parabolic trap whereas it is a free angular diffusion along the channel. Moreover, this indicates that the formal coupling which exists between these two coordinates in the Langevin equation, is not relevant in our experiment. So we can use this set-up in order to study SFD processes through the orthoradial diffusion of $N$ interacting particles.

\section{DIFFUSION OF INTERACTING PARTICLES IN A CIRCULAR CHANNEL}

We now consider rings of $N=12$ and $N=16$ interacting particles. These two systems were selected because in our channel geometry, these rings remain circular and do not present "zig-zag" configurations which could alter the SFD effects 13]. Furthermore, these rings also appear as outer shells in Wigner islands whose dynamics will be presented in a next paper 14].

The typical radial confining potentials for $N=12$ are shown on Fig. [3 As for $N=1$, they are parabolic and temperature independent and their stiffness increases as $V_{c}^{2}$. However we observe a stiffening with $N$, which is independently confirmed by the increase of the frequencies obtained from the fit of the radial m.s.d. by the expression 2 (Fig. 5). The frequencies $\omega \simeq \omega_{0}$ are about 30 $\mathrm{s}^{-1}$ for $N=12$ and 16 whereas they are about $20 \mathrm{~s}^{-1}$ for $N=1$. This increase of the frequencies with $N$ for a small number of balls is in accordance with the numerical study presented in Ref. 13. Consequently, the 1D char- 


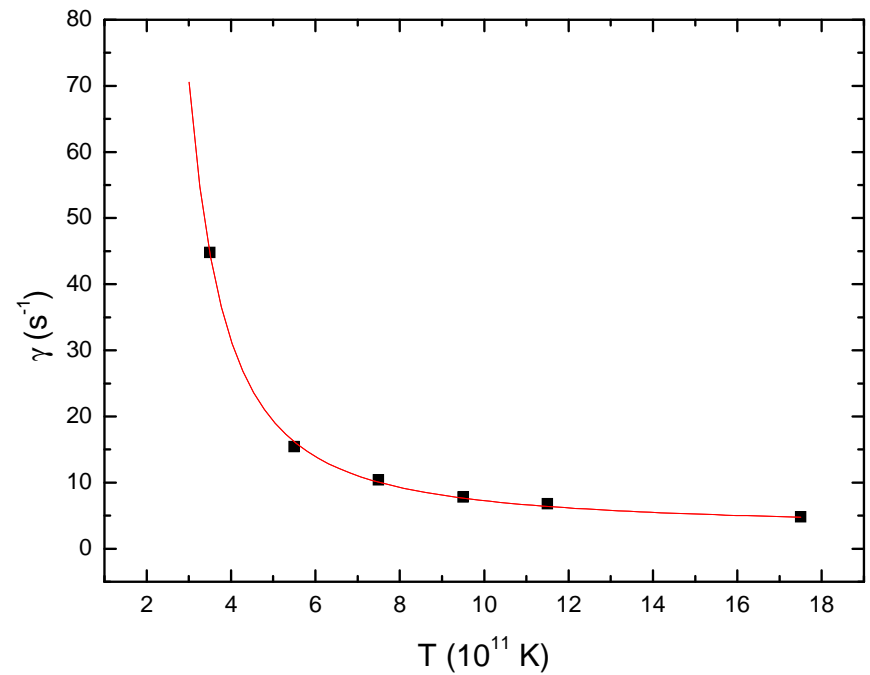

FIG. 6: Evolution with the temperature of the damping coefficient $\gamma$ for $N=12$ balls. Solid line shows the fit to the Guzman-Andrade law.

acter of the angular movement is amplified in the case of $N$ interacting balls. Moreover, this stiffening makes the parabolic modelization of the radial well all the more exact, and thus the determination of the damping coefficient $\gamma$ through the radial m.s.d. fits all the more accurate. As shown on Fig. [6] $\gamma$, which remains independent of the applied potential $V_{c}$ and of the number of balls, is a decreasing function of the temperature. Let us indicate that this last dependence, well fitted with the law $\gamma=A e^{B / T}$ suggests that our "thermal bath" looks more like a liquid than a gas for which $\gamma$ increases with $T$ [15]. We underline it does not imply that the electrostatic coupling between the balls is modulated by hydrodynamic effects as in Ref. [8].

Along the orthoradial direction, the distribution of the position of each ball is gaussian with a width proportional to $1 / N$. The amplitude of the displacement is small (about $2 \mathrm{~mm}$ ), so the curvature of the channel is not relevant. The variation with time of the orthoradial m.s.d is shown on Fig. [ 7 for $N=12$. As for the single-ball case, no oscillations are observed, confirming the complete decoupling between the radial and orthoradial movement. After a $t^{2}$ increase at short times, a fast transition to a subdiffusive behavior at long times is observed. However, and it is the strongest difference with the results presented for colloidal systems, this increase with time is slower than $t^{1 / 2}$ at long times. The same behavior is observed whatever $N$ and $V_{c}$. This continuous diffusion slowing down has never been mentioned previously, all the experiments on colloids concluding in the existence of only two distinct regimes : free diffusion characterized by a linear behavior at short times and a $t^{1 / 2}$ behavior at long times, attributed either to SFD or to hydrodynamics coupling effects. We can note also that, contrary to the studies on colloids, no clear linear regime is observed in our experiment. Indeed, the direct interaction

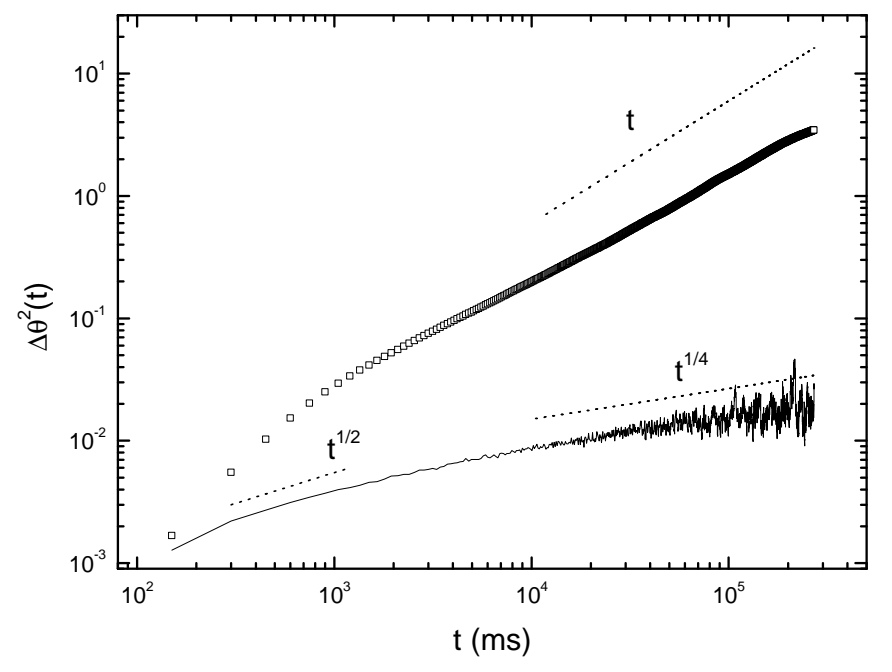

FIG. 7: Angular m.s.d. in log scale for a single ball ( $\square, V_{c}=$ $1000 \mathrm{~V}, T=15.1 \times 10^{11} \mathrm{~K}$ ) and a ball embedded in a 12 ball ring (solid line, $V_{c}=1000 \mathrm{~V}, T=3.5 \times 10^{11} \mathrm{~K}$ ). $\theta$ is in radians.

time which marks the switch between the normal and the subdiffusive regimes and qualitatively corresponds to the time when a particle "feels" the moving well around itself is quite short and of the same order as $\tau_{R}$ which characterizes the crossover between the $t^{2}$ and the linear free diffusion regimes.

Different reasons could explain these differences. The first one results from the characteristic times of the diffusion itself. In the colloidal systems, the direct interaction time (about $10 \mathrm{~s}$ ) appears on the reported data much larger than $\tau_{R}$ (which should be lower than 100 $\mathrm{ms}$ ), and then the linear regime can appear and be observed during one decade as in Ref. [9]. By contrast, in our case, the interaction is probably more long range, thus the presence of adjacent particles is more rapidly efficient and the crossover with the subdiffusive regime appears sooner. This assumption is confirmed by the decrease of the crossing time as the number of balls increases, in accordance with the enhancement of the repulsive interaction. Thus the linear regime is drastically reduced, hidden by the rapid emergence of the subdiffusive behavior. The second reason may be the limitation in time in the colloids experiments. In the latter, the subdiffusive behavior is put in evidence during another decade after the direct interaction time and is fitted as $t^{1 / 2}$. However, the m.s.d. time evolution in the figures presented Ref. 7, 9] exhibits ultimate points under the $t^{1 / 2}$ fit curves which suggests a slight slowing down beyond. This slowing down could be observed in our experiments and not in colloidal systems simply because they are performed up to a time of three order of magnitude higher than our direct interaction time which is roughly equal to $\tau_{R} \simeq 100 \mathrm{~ms}$. Longer recording in colloidal systems could give behaviors similar to ours.

Our results show unambiguously that the behaviors ob- 
served for colloidal systems are not due to hydrodynamic effects but describe real SFD. However, the fundamental origin of the observed slowing down is not clear yet. The theoretical results which predict $t^{1 / 2}$ at long times focus on infinite systems and have been performed either for hard-core interactions or for overdamped systems. Neither our Wigner rings (the oscillations observed in our radial m.s.d. prove moreover that our damping is weak) nor the colloidal systems correspond to these assumptions. So the usual theoretical predictions are not fully adapted to analyze our results which may not be discussed in this simple frame. We suggest that the observed slowing down could result from the periodicity of the system associated with an interaction that would be neither hard-core nor linear. Indeed, it has been proved that for hard-core interacting particles, the diffusion is normal for periodic systems [2]. On the other hand, for a linear interaction, the system of coupled Langevin equations can be solved in normal coordinates, and the contribution of the mode corresponding to the collective rotation gives a classical linear diffusion. Since neither our system nor the colloidal ones exhibit such a diffusion, this suggests that the distance dependence of the interaction is of high importance for periodic systems. These situations could introduce specific correlations between particles, not described in the usual models. It would be interesting to develop theoretical models including this new constraint.

\section{CONCLUSION}

Single file diffusion of electrostatically confined and interacting millimetric particles was studied. Their erratic motion conveyed through the mechanical shaking of the set-up was proved to be well described by the Langevin equation and thus to be Brownian. The characteristic parameters of the equation are well determined by fits of their radial mean square displacements.

Long time behavior of the angular mean square displacement was explored. We exhibit a subdiffusive behavior with a continuous slowing down that leads to a slower increase than the $t^{1 / 2}$ growth observed in colloidal systems. We suggest this might be a consequence of a non hard-core interaction in a periodic system.

\section{APPENDIX A: THE LANGEVIN APPROACH FOR A TRAPPED PARTICLE}

Experimental measurement of the time-dependent mean square displacement of a trapped particle requires to pay attention to the fact that the initial conditions of the measured trajectories are not equivalent, therefore the results are not exactly the same as the one usually presented in the theories. After a short summary of those usual results, we present what one should expect from an experimental determination such as ours.

We consider a $1 \mathrm{D}$ particle of mass $m$ and coordinate $x(t)$ trapped in a parabolic well of stiffness $K$ and immersed in a thermal bath characterized by a friction force $-\alpha \dot{x}$ and a rapidly fluctuating force $F$ which obeys $\langle F(t)\rangle=0$, where the notation \langle\rangle denotes an ensemble averaging. Newton's second law of motion gives :

$$
m \frac{d^{2} x}{d t^{2}}=-\alpha \frac{d x}{d t}-K x+F(t) .
$$

The major hypothesis of the Langevin equation is that $F$ is fluctuating with a characteristic time $\tau_{c}$ that is much lower than the time $\tau_{R}=\gamma^{-1}$ that characterizes the variation of the speed of the particle, where $\gamma=\alpha / m$ is the damping coefficient [16]. Then we can write $\left\langle F(t) F\left(t^{\prime}\right)\right\rangle=g \delta\left(t-t^{\prime}\right)$. Note that $\gamma$ has the same microscopic origin as the strength $F$, that's to say the numerous interactions with the particles of the thermal bath, then we should expect $\gamma$ to depend also on $g$, therefore on $T$.

If one considers a given initial condition $\left(x_{0}, v_{0}\right)$ and solves Eq. A1 one finds, in the case of a free particle $(K$ $=0)$, the following short and long time behaviors for the mean-square displacement $\Delta x^{2}(t)=\left\langle(x(t)-\langle x(t)\rangle)^{2}\right\rangle$ :

$$
\begin{aligned}
& \Delta x^{2}(t) \underset{t \rightarrow 0}{\sim} \frac{g}{3 m^{2}} t^{3}, \\
& \underset{t \rightarrow \infty}{\sim} 2 D t,
\end{aligned}
$$

where $D=\frac{g}{2 m^{2} \gamma^{2}}=\frac{k_{B} T}{m \gamma}$ is the diffusion constant.

For a trapped particle, the Langevin equation remains linear and is easily solved. In the case of weak friction $\left(\gamma<2 \omega_{0}=2 \sqrt{K / m}\right)$, one finds, for fixed $\left(x_{0}, v_{0}\right)$ initial conditions:

$$
\begin{aligned}
& \Delta x^{2}(t)=\frac{g}{2 m^{2} \gamma \omega^{2} \omega_{0}^{2}}\left[\omega^{2}+e^{-\gamma t}\right. \\
& \left.\quad \times\left(\frac{\gamma^{2}}{4} \cos (2 \omega t)-\frac{\gamma \omega}{2} \sin (2 \omega t)-\omega_{0}^{2}\right)\right],
\end{aligned}
$$

where $\omega^{2}=\omega_{0}^{2}-\frac{\gamma^{2}}{4}$.

In particular, for long times $\left(t \gg \tau_{R}\right)$,

$$
\Delta x^{2}(t) \sim \frac{g}{2 m^{2} \gamma \omega_{0}^{2}}=\frac{k_{B} T}{K} .
$$

However, in a majority of experiments, the averaging \langle\rangle is obtained by taking different trajectories considered as different realizations of the random theoretical trajectory. Those trajectories are obtained by shifting the initial time, that's to say, if $x(t)$ is the measured trajectory relatively to a given reference point, one considers the trajectories $x\left(t+t_{0}\right)-x\left(t_{0}\right)$, where each $t_{0}$ value gives a new trajectory.

For a free diffusion, this implies, since all points in space are equivalent, to take trajectories with the initial condition $x_{0}=0$. However, initial speed $v_{0}$ cannot be controlled and summation over the trajectories will also implies an averaging over the $v_{0}$ distribution. If the experiment has started for a time larger than $\tau_{R}$, 
we can consider that this distribution is the stationary one, thus the first moment of $v_{0}$ is zero and the second is $\frac{k_{B} T}{m}$. Note that this artefact cannot be avoided unless one is able to start all experiments with exactly the same conditions. Denoting $\langle\langle\rangle\rangle$ this new averaging, we find $\langle\langle x(t)\rangle\rangle=0$ and, noting that the $v_{0}$ and $F$ distributions are independent, the following behavior for the mean square displacement :

$$
\begin{aligned}
\Delta x^{2}(t) & \underset{t \rightarrow 0}{\sim} \frac{k_{B} T}{m} t^{2}, \\
& \underset{t \rightarrow \infty}{\sim} 2 D t .
\end{aligned}
$$

The main consequence is that $\Delta x^{2}(t)$ starts as $t^{2}$ for short times, and not as $t^{3}$ (Eq. A2). Long time behavior is not modified.

For a trapped diffusion, not all starting positions are equivalent and the averaging on the trajectories $x(t)-x_{0}$ necessarily implies averaging not only on the random force distribution but also on the initial condition $\left(x_{0}, v_{0}\right)$ distribution. Again, if the experiment has started for a time larger than $\tau_{R}$, we can consider that this distribution is the stationary one, then that the first moment of $x_{0}$ is zero and the second is $\frac{k_{B} T}{K}$.

We find that $\left\langle\left\langle x(t)-x_{0}\right\rangle\right\rangle=0$ and

$$
\begin{aligned}
& \Delta x^{2}(t)=2\left\langle\left\langle x_{0}^{2}\right\rangle\right\rangle \\
& \times\left[1-e^{-\gamma^{\prime} t}\left(\cos (\omega t)+\frac{\gamma^{\prime}}{\omega} \sin (\omega t)\right)\right] .
\end{aligned}
$$

In particular :

$$
\Delta x^{2} \underset{t \rightarrow 0}{\sim} \frac{k_{B} T}{m} t^{2} .
$$

Short time behavior is thus independent from the well and similar to the free diffusion case. Note also that the infinite time limit $\frac{2 k_{B} T}{K}$ is twice the theoretical value in the case of identical initial condition trajectories.
[1] T. E. Harris, J. Appl. Prob. 2, 323 (1965).

[2] H. van Beijeren, K. W. Kehr, and R. Kutner, Phys. Rev. B 28, 5711 (1983).

[3] M. Kollmann, Phys. Rev. Lett. 90, 180602 (2003).

[4] S. S. Nivarthi, A. V. McCormick, and H. T. Davis, Chem. Phys. Lett. 229, 297 (1994).

[5] K. Hahn and J. Kärger, J. Phys. Chem. B 102, 5766 (1998), and references therein.

[6] H. Jobic, K. Hahn, J. Kärger, M. Bee, A. Tuel, M. Noack, I. Girnus, and G. J. Kearley, J. Phys. Chem. B 101, 5834 (1997).

[7] Q. H. Wei, C. Bechinger, and P. Leiderer, Science 287, 625 (2000).

[8] B. Lin, B. Cui, J.-H. Lee, and J. Yu, Europhys. Lett. 57, 724 (2002).

[9] C. Lutz, M. Kollmann, P. Leiderer, and C. Bechinger, J. Phys.: Condens. Matt. 16, S4075 (2004).

[10] P. Galatola, G. Coupier, M. Saint Jean, J.-B. Fournier, and C. Guthmann, cond-mat/0602272.

[11] G. Coupier, C. Guthmann, Y. Noat, and M. Saint Jean, Phys. Rev. E 71, 046105 (2005).

[12] C. Aslangul, in Anomalous diffusion. From basis to applications. Proceedings of the 11th Max Born Symposium
(1999), p. 326.

[13] I. V. Schweigert, V. A. Schweigert, and F. M. Peeters, Phys. Rev. B 54, 10827 (1996).

[14] G. Coupier, M. Saint Jean, and C. Guthmann, to be published.

[15] The law between the viscosity of a liquid and the temperature is known as the Guzman-Andrade law. See for instance E. Guyon, J.-P. Hulin, and L. Petit, Hydrodynamique physique (EDP Sciences / CNRS Editions, 2001) for the differences between gas and liquids in the temperature dependence of the viscosity. Note however that the explored temperature range is not large enough to conclude definitively.

[16] Let us indicate that, in the light of our experiment the Langevin formalism seems to apply even if the time $\tau_{R}$ characterizing the relaxation process is not that much larger than the time $\tau_{c}$ linked with the surrounding thermal bath. The latter can be evaluated from the average frequency of our "white noise", which indeed is white within the range $0-200 \mathrm{~Hz}$. So we have $\tau_{c} \simeq 10 \mathrm{~ms}$, to be compared with $\tau_{R} \simeq 100 \mathrm{~ms}$. 